\title{
Theoretical treatments of fusion processes in collisions of weakly bound nuclei* $^{*}$
}

\author{
L.F. Canto ${ }^{\mathrm{a}}$, R. Donangelo ${ }^{\mathrm{a}}$ and M.S. Hussein ${ }^{\mathrm{b}}$ \\ ${ }^{a}$ Instituto de Física, Universidade Federal do Rio de Janeiro, Cidade Universitária, CT \\ Bloco A, 21941-972 Rio de Janeiro, RJ, Brasil \\ bInstituto de Física, Universidade de São Paulo, C.P. 66318, 05389-970, São Paulo, SP, \\ Brasil
}

We review the theoretical methods to evaluate fusion cross sections in collisions of weakly bound nuclei. We point out that in such collisions the coupling to the breakup channel leads to the appearance of different fusion processes. The extension of the coupledchannel method to coupling with the continuum is the most successful treatment for these collisions. However, evaluating separate cross section for each fusion process remains a very hard task.

\section{INTRODUCTION}

The theoretical description of the fusion of nuclei has so far relied on a many-degrees-offreedoms tunneling formalism referred to as the Coupled Channels Theory. In the fusion of stable nuclei the channels considered correspond to bound states in the participating nuclei. When weakly bound nuclei fuse, the coupling to continuum channels becomes important and one resorts to by and large intuitive means to take these into account [1]. If fusion occurs above the Coulomb barrier, quantum tunneling is less important and one uses geometrical arguments to consider these couplings. In fact this is also done in the description of the fusion of fullerene molecules [2], important in the development of strong nano-tubes. At near or sub-barrier energies, tunneling becomes a dominant feature of the fusion of nuclei and considering the coupling to the continuum or breakup channels has been a challenge to theorists. The reason being the need for a full three-body model for tunneling that describes both the complete fusion as well as the incomplete fusion of one of the fragments when breakup takes place. In this three-body model the interactions have to be complex. So far no such complete theory is practically developed, although serious attempts towards this goal have been made [3]. Less ambitious endeavours based on a discretized Continuum within a basically extended two-body model have been extensively used. This is the Continuum Discretized Coupled Channels (CDCC) method. Variants of this method have also been pursued especially in the fusion of radioactive nuclei. In this contribution we present a short account of some of these theoretical attempts to treat the quantum tunneling of fragile objects.

\footnotetext{
*This work was supported in part by the CNPq, the FAPERJ/CNPq(PRONEX) and the FAPESP.
} 


\section{THE FUSION CROSS SECTION IN COLLISIONS OF STRONGLY BOUND NUCLEI}

Let us first consider the fusion cross section in potential scattering, where the components of the projectile-target separation vector are the only degrees of freedom taken into account. The collision dynamics is described by the Hamiltonian

$H=T+U$,

where $T$ is the kinetic energy operator and $U$ is the optical potential. In typical situations, one has

$U=V+i\left(W^{F}+W^{D}\right)$

where $W^{F}$ and $W^{D}$ are absorptive potentials, accounting for the flux lost to fusion and to peripheral processes, respectively. The scattering state $\left|\psi^{(+)}\right\rangle$is obtained solving the Schrödinger equation with scattering boundary condition and the fusion cross section is

$\sigma_{F}=-\frac{k}{E}\left\langle\psi^{(+)}\left|W^{F}\right| \psi^{(+)}\right\rangle$

Above, $E$ is the collision energy and $k$ is the wave number associated to it.

In multi-channel scattering the problem is more complicated since the Hamiltonian depends also on intrinsic degrees of freedom. The scattering wave function is expanded in eigenstates of the intrinsic Hamiltonian, $|\alpha\rangle$, with eigenvalues $\varepsilon_{\alpha}$, in the form

$$
\left.\left|\Psi^{(+)}\right\rangle=\sum_{\alpha}\left|\psi_{\alpha}^{(+)}\right\rangle \mid \alpha\right)
$$

and the channel wave functions, $\psi_{\alpha}^{(+)}$, are given by the coupled-channel equations,

$$
\left[E_{\alpha}-V_{\alpha}-W_{\alpha}\right] \psi_{\alpha}^{(+)}=\sum_{\beta(\neq \alpha)} U_{\alpha, \beta} \psi_{\beta}^{(+)} ; \quad \alpha, \beta=0,1, \ldots
$$

Above, $E_{\alpha}=E-\varepsilon_{\alpha}$,

$V_{\alpha}+W_{\alpha}=(\alpha|U| \alpha) \quad$ and $\quad U_{\alpha, \beta}=(\alpha|U| \beta)$.

If all relevant direct channels are included in the expansion, the imaginary potential $W_{\alpha}$ represents exclusively fusion absorption. If the off-diagonal part of the interaction, $U_{\alpha, \beta}$, is real, as frequently is the case, the fusion cross section can be written

$\sigma_{F}=-\frac{k}{E} \sum_{\alpha}\left\langle\psi_{\alpha}^{(+)}\left|W^{F}\right| \psi_{\alpha}^{(+)}\right\rangle$.

When the channel coupling interaction is complex, off-diagonal terms should also be included in the summation above.

It is well known that channel coupling leads to a strong enhancement of the fusion cross section at sub-barrier energies [4]. That is, the sum of the contributions from all channels is larger than the fusion cross section when the coupling is switched off. However, it is important to investigate the effect of the coupling on the fusion through the 
elastic channel alone, $\sigma_{F, 0}$. For this purpose it is convenient to introduce the polarization potential. Although its calculation may be difficult, it provides a convenient language for the discussion. This potential is defined by the condition that the same elastic wave function $\psi_{0}^{(+)}$of the Coupled-channel equations be obtained from a potential scattering equation (eq. (4) with the RHS set equal to 0), with the replacements

$$
\begin{array}{cc}
V_{0} & \rightarrow V_{0}+V_{p o l} \\
W_{0} & \rightarrow W_{0}+W_{p o l} .
\end{array}
$$

The inclusion of $W_{\text {pol }}$ always reduces the fusion cross section, since it leads to absorption of the incident flux before it reaches the region where fusion takes place. The real part of the potential may lead to additional fusion suppression, if it is repulsive. However, if it is attractive, it reduces the barrier height and thus enhances the fusion cross section. In this case, there is a competition between the real and the imaginary parts of the polarization potential and the net result depends on the details of that potential.

\section{FUSION OF WEAKLY BOUND NUCLEI}

Collisions of weakly bound nuclei are much more complicated since the elastic channel couples strongly with continuum states. In this case, the channel label becomes continuous and there is an infinite number of coupled channels, even with truncation at low values of the excitation energy. A theory including continuum coupling and describing the various fusion processes is very hard to develop. An important feature of these collisions is that the breakup mechanism may give rise to two different types of fusion. Complete fusion (CF), when the whole masses of the projectile and the target are contained in the compound nucleus, and incomplete fusion (ICF), when some nucleons move out of the interaction region before the formation of the compound nucleus. An additional complication is that $\mathrm{CF}$ can take place in a single step or in a sequential way, as the fragments of the broken projectile are successively absorbed by the target. These mechanisms are called Direct (DCF) and sequential (SCF) complete fusion, respectively. The sum of CF and ICF is termed total fusion ( $\mathrm{TF})$.

In this section, we summarize some of the attempts available in the literature, with emphasis on the Continuum Discretized Coupled Channels method (CDCC).

\subsection{Early models}

The earliest calculation of fusion cross sections in collisions of weakly bound nuclei were carried out with schematic models, based on drastic approximations. The static effects of the halo could easily be taken into account through the use of larger diffusivities in the real potential. However, the inclusion of dynamic effects is a much harder task, since it involves the coupling with continuum states.

In the first calculations, coupled channel effects were mocked up by an approximate polarization potential. Since the adopted approximations led to a purely imaginary potential, changes in the barrier hight were simulated by a shift in the collision energy. These works found that the CF cross section was hindered around the barrier energy [ 6. Soon after these works, a calculation adopting the opposite view was performed by Dasso and Vitturi [7]. These authors performed coupled channel calculations in a simple 
model in which the continuum was represented by a bound effective channel. The CF cross section was then obtained from eq. (6), with the exclusion of the effective channel from the summation. However, in this model important properties of the continuum were not properly described. In opposition to the previous results, they obtained a strongly enhanced CF cross section.

Since these calculations were based on very schematic models, using different approximations, it is not surprising that they led to conflicting conclusions. It is clear that a reliable conclusion about the dynamic effects of the coupling requires the use of more realistic models.

\subsection{The CDCC method and its applications}

The CDCC method consists of replacing the continuous part of the projectile's spectrum by a finite number of discrete states. This can be done in different ways [5]. The most frequent approach is to divide the continuum in bins, distributed up to some cut-off energy $E_{\text {max }}$, and to build wave packets within each bin. The wave packets are superpositions of energy eigenstates $f_{\alpha}(k, r)$, where $k$ is the wave number associated with the relative motion of the projectile's fragments and $\alpha$ stands for the remaining projectile's quantum numbers. One common option is to use $N$ bins of equal size, $\Delta$, in momentum space, distributed between 0 and $k_{\max }=N \Delta$. In this case, the $i^{t h}$ discrete state with quantum numbers $\alpha$ is

$u_{\alpha, i}(r)=\int_{k_{i}-\Delta / 2}^{k_{i}+\Delta / 2} g_{\alpha}\left(k-k_{i}\right) f_{\alpha}(k, r) d k$.

The particular shapes of the wave packets do not significantly affect the results. However, they should be chosen such that the states $u_{\alpha, i}(r)$ form an orthonormal set. In this way the problem is reduced to a set of coupled-channel equations involving the elastic, the excited bound channels and $N$ discretized channels for each $\alpha$.

An example of the application of this method to the ${ }^{8} \mathrm{~B}$ breakup process may be found in ref. [ 8]. The comparison of the full CDCC calculations with other simplified calculations shows the importance of higher-order couplings, and also of the couplings between continuum states.

This last ingredient, continuum-continuum couplings, had not been included in the calculations of the fusion cross section for the ${ }^{11} \mathrm{Be}+{ }^{208} \mathrm{~Pb}$ collision presented in ref. [ 9. Their results are shown in figure 1, in comparison with predictions of a bare potential (channel-coupling switched off). Within those conditions, the CF cross section is strongly enhanced at low energies and slightly hindered at high energies. The transition between these behaviors occurs at an energy $E_{t r}>V_{B}$. Thus, this study leads to enhancement of the $\mathrm{CF}$ cross section in the barrier region.

The effect of the continuum-continuum couplings on this reaction was investigated by Diaz-Torres and Thompson [10]. Their results are shown in figure 2, Continuumcontinuum couplings were not included in the CDCC calculations presented in (a) and were included in the ones shown in (b). In (a), the results are similar to those of ref. [9] while in (b) they are rather different. Both the $\mathrm{CF}$ and $\mathrm{TF}$ cross sections are much smaller than those obtained without continuum-continuum couplings. In this case, the CF cross section is suppressed in the barrier region, with $E_{t r}$ falling below the Coulomb barrier. It 


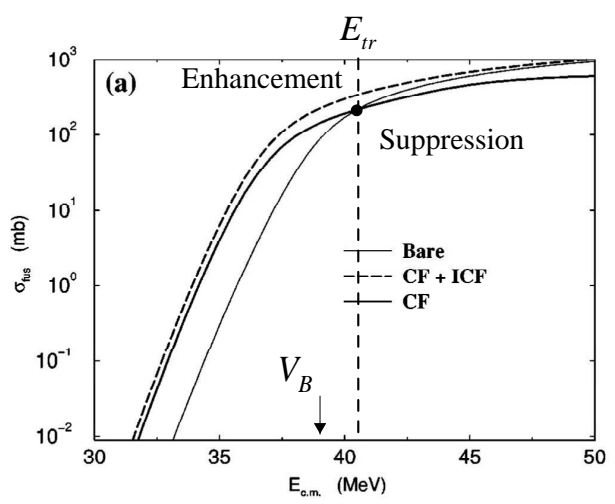

Figure 1. CDCC calculation of the fusion cross sections without continuum-continuum couplings (figure from ref. [9])

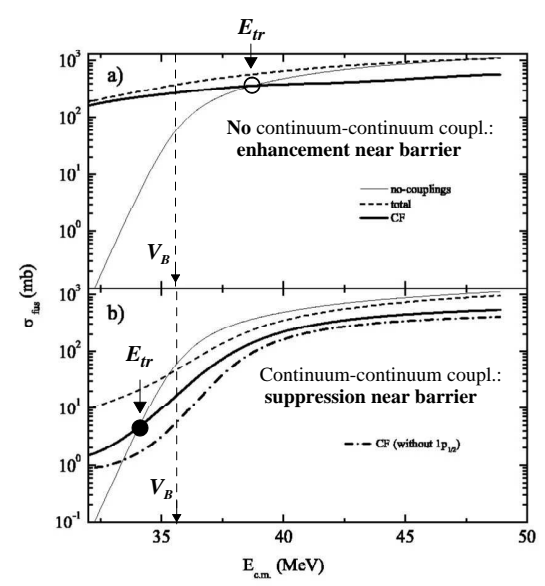

Figure 2. CDCC calculation of the fusion cross sections with and without continuum-continuum couplings (figure from ref. [10]).

should be remarked that neither of these works take into account the sequential capture of all fragments, and thus they provide only a lower limit for the CF cross section.

Intuitively, the reduction of the fusion cross section arising from couplings among many channels in the continuum sounds reasonable. It is, however, of interest to prove this formally. One can formulate the problem using Feshbach theory and find a way of eliminating the continuum-continuum coupling terms. What one finds is a set of coupled channels equations of the type:

$$
\begin{aligned}
& \left(E_{0}-H_{0}\right) \Psi_{0}=\sum_{c} V_{0 c} \Psi_{c} \\
& \left(E_{c}-H_{c}\right) \Psi_{c}=F_{c 0} \Psi_{0}
\end{aligned}
$$

where $V$ and $F$ are different operators. Now we know from compound nucleus theory that if this happens (the c-channels are closed here) then time reversal is violated according to the definition of Wigner of time reversal invariance [11]. If time reversal is violated one has irreversibility. However, what we are interested here is the so-called statistical mechanics irreversibility. Of course, in order for this to happen, one has to resort to coarse graining the continuum-continuum couplings (energy-averaging) as it has been done extensively in the past in the treatment of Deep Inelastic Collisions [12]. This may indeed be happening in the actual calculations using the CDCC method.

New calculations employing the CDCC method to determine the effect of the breakup channel on the total fusion cross section in reactions induced by ${ }^{6,7} \mathrm{Li}$ projectiles were performed more recently [13]. The main difference between these calculations and those of ref. [10] is in the absorptive interaction. Ref. [13] uses a projectile-target imaginary potential while ref. [13] considers absorption of each projectile's fragment separately. In this way, only the TF cross section is evaluated. It was found through those calculations [ 
13] that the presence of the breakup channel enhances the total (complete + incomplete) fusion cross section in the region around the Coulomb barrier, and it has very little effect at higher energies. It was further observed that the effect depended on the target size, as expected, being more marked in the case of a ${ }^{209} \mathrm{Bi}$ than for ${ }^{59} \mathrm{Co}$. The differences in total fusion cross section between the two Lithium projectiles may be attributed to the coupling to the breakup channel in the case of ${ }^{59} \mathrm{Co}$, but not for the ${ }^{209} \mathrm{Bi}$ target.

One should also mention the combined CDCC-Dynamic Polarization Potential (DPP) approach introduced by Keeley, Rusek and collaborators [14, 15]. The procedure consists in first performing CDCC calculations to describe the elastic scattering data for the system under consideration, and from the calculations extract the corresponding polarization potentials. The fusion cross sections are then obtained using a simple barrier penetration calculation, where the barrier is given by the addition of the real parts of the optical potential and the polarization potential.

This CDCC-DPP method was employed to extract the effect of the ${ }^{6} \mathrm{He}$ dipole polarizability on its elastic scattering on ${ }^{208} \mathrm{~Pb}$, and its correspondent effect on the fusion cross section for this reaction. A comparison of the theoretical fusion cross section of the projectiles ${ }^{6} \mathrm{He}$ and ${ }^{6} \mathrm{Li}$ incident on ${ }^{208} \mathrm{~Pb}$ is shown in fig.3. As there is no available data for these systems, the calculations are compared with data for the ${ }^{6} \mathrm{He}$ on ${ }^{209} \mathrm{Bi}$ fusion cross section. The figure shows results for these projectiles with and without coupling to the breakup channel. When this coupling is left out (only static effects), the cross section for ${ }^{6} \mathrm{He}$ is much larger than that for ${ }^{6} \mathrm{Li}$, owing the ${ }^{6} \mathrm{He}$ neutron halo. When breakup couplings are taken into account, repulsive potentials reduce the cross sections. Since this effect is stronger for ${ }^{6} \mathrm{He}$, the static effect of the halo is cancelled and the fusion cross section for the two projectiles are similar. The CDCC cross section for ${ }^{6} \mathrm{He}$ projectiles is consistent with the data.

The breakup of the ${ }^{6} \mathrm{He}$ and ${ }^{6} \mathrm{Li}$ projectiles proceeds according to different mechanisms. In the case of ${ }^{6} \mathrm{He}$, the $E 1$ transitions to the continuum dominate. As the ${ }^{4} \mathrm{He}$ and ${ }^{2} \mathrm{H}$ fragments of ${ }^{6} \mathrm{Li}$ have the same charge/mass ratios, these transitions do not play a role in the case of ${ }^{6} \mathrm{Li}$, which breakup is thus produced by quadrupole couplings, mainly driven by nuclear forces.

\subsection{Other approaches}

Besides the quantal coupled-channels procedures mentioned above, other alternative methods have been employed to describe the fusion and breakup processes in reactions induced by weakly bound projectiles. Recently, Hagino and collaborators have developed a classical trajectory method, in which the classical trajectories for the target and the two projectile fragments are evaluated for a Monte Carlo sampling of initial conditions [16]. This method automatically separates the complete and incomplete fusion cross sections, so it does not suffer from the deficiency of the CDCC calculations presented in [10] which count the sequential fusion of all fragments as an incomplete, rather than a complete fusion process. In fact, according to these classical calculations, the cross section for the capture of all fragments represents an important contribution to the complete fusion cross section. The results of these calculations are in reasonable agreement with the experimental data at energies above the Coulomb barrier. However, as they do not include tunneling effects, they cannot be employed at the underbarrier energies where much of the interest in fusion 


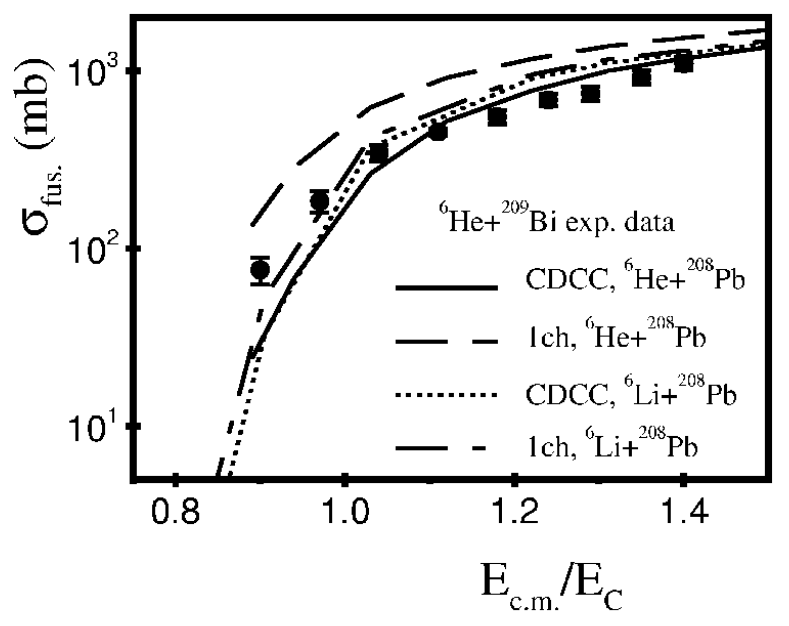

Figure 3. CDCC and single-channel calculations of fusion cross sections for the ${ }^{6} \mathrm{He}+$ $208 \mathrm{~Pb}$ and ${ }^{6} \mathrm{Li}+{ }^{208} \mathrm{~Pb}$ systems. Data for ${ }^{6} \mathrm{He}+209 \mathrm{Bi}$ are also shown (figure from ref. [ 15]).

induced by weakly bound projectiles lies.

A time-dependent description of the collision process was developed to study fusion reactions induced by halo nuclei [17]. In this approach the system is described as a three-body system, composed of the target, the projectile core and the halo nucleon. This three-body reaction problem is described in terms of the time evolution of a wave packet. The initial wave packet is taken to be the product of an incoming wave for the relative motion between the centers of mass of the projectile and target nuclei, multiplied by a Gaussian wave packet representing the initial configuration of the projectile fragments. The evolution of this wave packet is treated through the standard time-dependent Schrödinger equation approach, which although requiring long calculation times, is simple to program. The fusion probability is calculated through an energy projection procedure. This approach has been applied to several systems [18]. In the case of neutron halos, it is found that the effect of the halo hinders the fusion cross section.

Since semiclassical calculations of continuum discretization coupled-channels have been successfully applied to the description of the breakup of weakly bound projectiles [ 19], they should be extendable to the treatment of fusion reactions. The breakup amplitudes calculated with the semiclassical calculations would determine the initial conditions for the study of the fragments. Such a procedure would have the advantage of automatically separating the motion of all fragments, thus separating the sequential fusion contribution to complete fusion from the incomplete fusion process, which was one of the difficulties facing the quantum mechanical CDCC calculations. A preliminay calculation of the CF cross section in a schematic two-channel model has recently been performed [20] and the results were compared with results of the coupled channel method. The agreement was 
good.

\section{SUMMARY}

In this paper we have presented a brief review of the theoretical methods to describe fusion reactions with weakly bound nuclei. We pointed out that these reactions involve the coupling to continuum states, which is very hard to describe. In this way, simple models using drastic approximations can hardly provide a reasonable description of the fusion processes. The most successfull calculations performed so far are based on the CDCC method. The major lesson we have learnt from them is that continuum-continuum couplings play an essential role in the fusion processes, reducing substantially the CF cross section. This result arises from the statistical irreversibility of transitions to the continuum. We have pointed out that the presently available CDCC calculations have the shortcoming of not providing separate descriptions of the various fusion processes following breakup. This is a very hard task, which might be handled in a better way in classical and semiclassical models.

\section{REFERENCES}

1. L.F. Canto, P.R.S. Gomes, R. Donangelo and M.S. Hussein, Phys. Rep. 424 (2006) 1.

2. E.E. Campbell, Fullerene Collision Reactions, Kluwer Academic Publishers, Dordrecht, the Netherlands (2003).

3. T. Matsumoto, E. Hiyama, K. Ogata, Y. Iseri, M. Kamimura, S. Chiba and M. Yahiro, Phys. Rev. C70 (2004) 061601.

4. C.H. Dasso, S. Landowne and Aa. Winther, Nucl. Phys. A405 (1983) 381; A407 (1983)221; W. Reisdorf et al., Nucl. Phys. A614 (1997) 112.

5. see e.g. N. Austern et al., Phys. Rep. 154 (1987) 125 and references therein; F. PéresBernal et al., Phys. Rev. A63 (2001) 052111.

6. M.S. Hussein, M.P. Pato, L.F. Canto and R. Donangelo, Phys. Rev. C46 (1992) 377; N. Takigawa, M. Kuratani and H. Sagawa, Phys. Rev. C47 (1993) R2470.

7. C.H. Dasso and A. Vitturi, Phys. Rev. C50 (1994) R12.

8. J.A. Tostevin, F.M. Nunes, and I.J. Thompson, Phys. Rev. C 63 (2001) 024617.

9. K. Hagino, A. Vitturi, C.H. Dasso and S. Lenzi, Phys. Rev. C61 (2000) 037602.

10. A. Diaz-Torres and I.J. Thompson, Phys. Rev. C65 (2002) 024606.

11. H. Feshbach, M.S. Hussein and A.K. Kerman, Z. Phys. A351 (1995) 133.

12. C.M. Ko, D. Agassi and H.A. Weidenmüller, Ann. Phys. 117 (1979) 237.

13. A. Diaz-Torres, I.J. Thompson and C. Beck, Phys. Rev. C 68 (2003) 044607.

14. N. Keeley and K. Rusek, Phys. Lett. B 427 (1998) 1; N. Keeley, K.W. Kemper and K. Rusek, Phys. Rev. C65 (2001) 014601; ibid. Phys. Rev. C66 (2003) 044605(R);

15. K. Rusek, N. Keeley, K.W. Kemper and R. Raabe, Phys. Rev. C67 (2003) 041604(R).

16. K. Hagino, M. Dasgupta and D.J. Hinde, Nucl. Phys. A 738 (2004) 475.

17. K. Yabana, M. Ito, M. Kobayashi, M. Ueda and T. Nakatsukasa, Nucl. Phys. A 738 (2004) 303.

18. M. Ito, K. Yabana, T. Nakatsukasa and M. Ueda, arXiv:nucl-th/0506073.

19. H.D. Marta, L.F. Canto, R. Donangelo and P. Lotti, Phys. Rev. C 66 (2002) 83.

20. L.F.Canto, H.D. Marta and R. Donangelo, Phys. Rev. C 73 (2006) 034608. 